\title{
Measuring the Decoupling Progress in Developed and Developing Countries
}

\author{
Heming Wang ${ }^{1, a^{*}}$, Shen Zhao ${ }^{1, b}$, Yao Wei ${ }^{1, c}$, Qiang Yue ${ }^{1, d}$, and Tao Du ${ }^{1, e}$ \\ ${ }^{1}$ State Environmental Protection Key Laboratory of Eco-Industry, Northeastern University, China \\ awanghm@mail.neu.edu.cn, bzhaoshen_2016@hotmail.com, cweiyao_2018@hotmail.com,

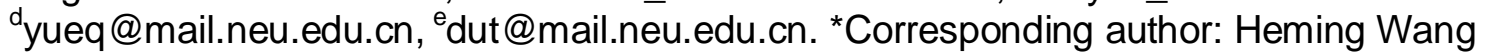 \\ * The corresponding author
}

\section{Keywords: Resource use; Economic Growth; Decoupling analysis; Sustainable development}

\begin{abstract}
The relationship between economic development and the ecological environment is the key to sustainable development of human society. With the rapid economic development, the demand for resources has increased dramatically, which has led to problems such as depletion of resources and environmental pollution. Therefore, the relationship between resource use and economic growth needs to be studied to formulate corresponding resource use policies to facilitate the sustainable development of human society. This paper uses the resource decoupling indicators to study the decoupling condition of domestic material consumption (DMC) and material footprint (MF) indicators of three BRICS countries and three OECD countries from 1990 to 2015. Resources include biomass, non-metallic minerals, fossil fuels, and metal ores. Based on the results from these cases, we find that developed countries have a better decoupling condition than developing countries in the DMC level, but there is not much difference between developed and developing countries at the MF level. The developing countries' decoupling condition of MF is better than that of DMC, while the developed countries' decoupling condition is opposite. This study also emphasizes the importance of developing decoupling policies by selecting an appropriate resource use indicator.
\end{abstract}

\section{Introduction}

The relationship between economic development and the ecological environment is the key to sustainable development[1-2]. The rapid economic growth and resource use has caused various environmental problems[3-5]. There is an urgent need to study their relationship to realize the sustainable development of human society. Under normal circumstances, the resources use is always "linked" with the economy, and even they are synchronous growing. Therefore, the decoupling problem becomes crucial to the implementation of the sustainable development strategy. Decoupling means that the growth rate of the environmental impact in a certain period is less than its' economic growth rate (relative decoupling) or the environmental impact keeps constant or declines (absolute decoupling). It is important to investigate the quantitative relationship between them to establish a more scientific and rational decoupling evaluation index. The curve describing the development trend of resources to developed countries is called "environmental mountain (EM)"[6]. It is better for developing countries to tunnel through the EM to achieve economic growth while paying fewer environmental cost [7-9].

This article uses decoupling indicators for resource use from the well-known IPAT equation[6, 8]. By using the DMC and MF indicators, we compared the biomass, non-metallic minerals, fossil fuels, and metal ores' decoupling condition of three BRICS (Brazil, India, and China) countries and three OECD countries (Australia, Japan, and USA) from 1990 to 2015. Based on the results from these case studies, we provide some development suggestion to reduce resource use and figure out insights for developing countries to achieve resource decoupling goals.

\section{Methods and data}

The decoupling indicator (resource use) is the basis for the decoupling analysis, and it can be expressed mathematically as 
$\mathrm{D}=\frac{t}{g}(1+g)$

Where $D$ is the decoupling indicator for resource use, $G$ is the GDP growth rate, and $t$ is the rate of decline in resource use per unit of GDP (when $t$ is positive, the use of resources per unit of GDP is declining).

The derived equation for resource decoupling analysis can be expressed as

$\log \left(\mathrm{I}_{\mathrm{n}} / \mathrm{I}_{0}\right)=\mathrm{n} \times \log [1+\mathrm{g} \times(1-\mathrm{D})]$

Where, $I_{0}$-resource use in the base year; $I_{n}$-resource use in the nth year after the base year; $n$ - ordinal number of years after the base year.

Two parameters $g$ and $t$ represent the changes in resource use, where $g$ is the geometric average growth rate of GDP, and $t$ is the geometric average rate of decline in the use of unit GDP. The parameters $g$ and $t$ are calculated by the following equations as

$$
\begin{gathered}
\mathrm{g}=\left(G_{n} / G_{0}\right)^{\frac{1}{n}}-1 \\
\mathrm{t}=1-\left(T_{n} / T_{0}\right)^{\frac{1}{n}}
\end{gathered}
$$

Here, $T_{0}$ and $G_{0}$ are the unit GDP resource use and GDP in the base year respectively, and $T_{n}$ and $G_{n}$ are the unit GDP resource use and GDP after the $\mathrm{n}^{\text {th }}$ year respectively.

The Material flow and GDP data are from Global Material Flows Database [10].

\section{Results and Analysis}

Fig. 1 and Fig. 2 show the decoupling results of resources' DMC and MF in China during 1990-2015, respectively. Generally, China has achieved relative decoupling goal during 1990-2015. From the viewpoint of DMC, biomass enjoy the best decoupling condition whose D value is 0.7 approximately, and metal ores' condition is worst whose $\mathrm{D}$ value is nearly 0 in 2015 . The decoupling growth tendency of China's MF is similar to that of China's DMC, but their condition is a little different. During 2009-2015, metal ores is not decoupled from GDP, which means the growth rate of metal ores is higher than that of GDP.

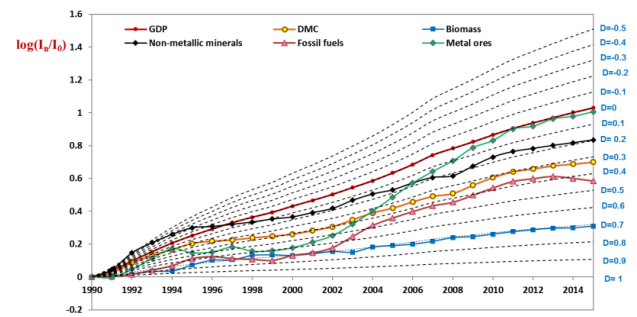

Figure 1. The decoupling trend of DMC in China, 1990-2015.

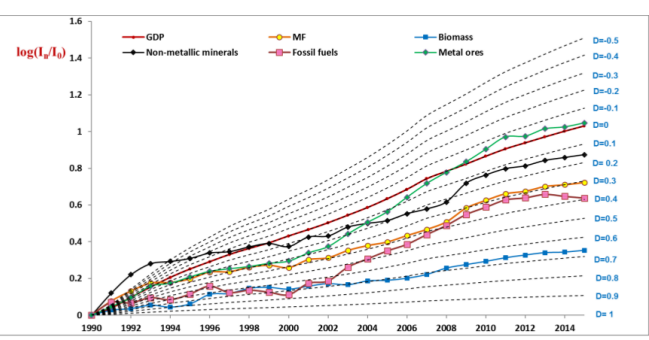

Figure 2. The decoupling trend of MF in China, 1990-2015.

Fig. 3 and Fig. 4 show the decoupling results of resources' DMC and MF in Brazil during 1990-2015, respectively. The decoupling condition of Brazil is the worst in our study, and three kinds of resources' DMC and MF are not decoupled from GDP in 2015. Metal ores are the only resources which achieve a decoupling goal during 1990-2015. With the GDP's decreasing during 2013-2015, Brazil's DMC and MF is increasing and led the D value to be lower than before. In addition, the growth tendency of $\mathrm{D}$ values in Brazil is not smooth like other countries, especially for that metal ores' DMC/MF and fossil fuels' MF show an unstable tendency.

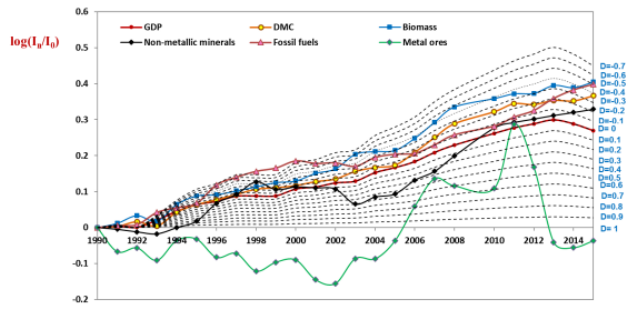

Figure 3. The decoupling

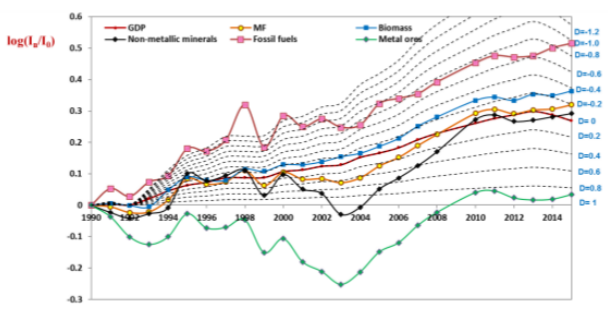

Figure 4. The decoupling 
trend of DMC in Brazil, 1990-2015. $\quad$ trend of MF in Brazil, 1990-2015.

Fig.5 and Fig.6 show the decoupling results of resources' DMC and MF in India during 1990-2015, respectively. The decoupling growth tendency in India is similar to that in China, and India has achieved relative decoupling goal during 1990-2015 generally. Like the results in China, biomass has the best decoupling condition while metal ores have the worst from the viewpoint of resources, and the decoupling growth tendency is also smooth and continuous. However, metal ores' DMC is not decoupling in India, and their tendency is unstable during 1990-2015.

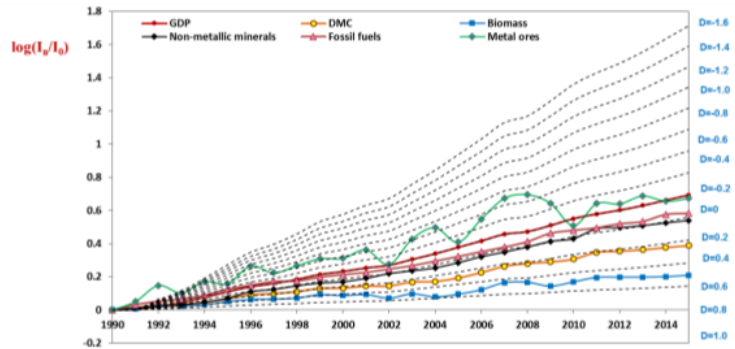

Figure 5. The decoupling trend of DMC in India, 1990-2015.

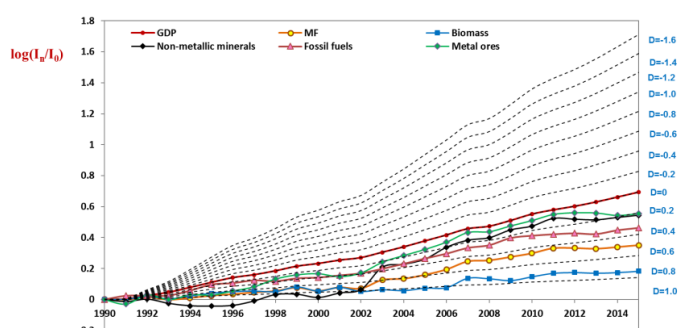

Figure 6. The decoupling trend of MF in India, 1990-2015.

Fig. 7 and Fig. 8 show the decoupling results of resources' DMC and MF in Australia during 1990-2015, respectively. From the DMC viewpoint, the decoupling conditions of all resources are relative decoupling. Their D value is increasing significantly during 2000-2015, and tending to 0.6 finally. The decoupling condition of MF is also relative decoupling, and the biomass' $\mathrm{D}$ value is 0.6 while other resources are tending to 0.2 in 2015. The characteristic of MF's decoupling condition in Australia is unstable but synchronous growth, especially during 1992-1995 and 2002-2007.

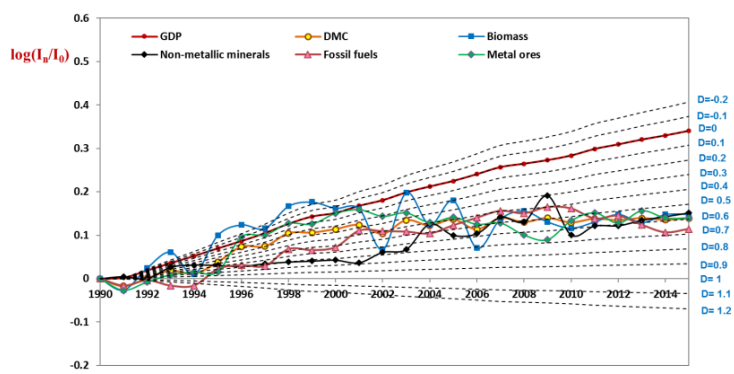

Figure 7. The decoupling trend of DMC in Australia, 1990-2015.

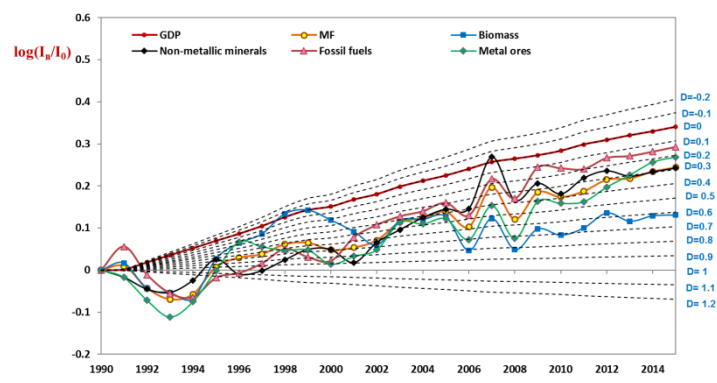

Figure 8. The decoupling trend of MF in Australia, 1990-2015.

Fig. 9 and Fig.10 show the decoupling results of resources' DMC and MF in Japan during 1990-2015, respectively. The decoupling condition of Japan's DMC is the best in our study. During 1990-2015, biomass, non-metallic minerals and metal ores are all absolute decoupling. Fossil fuels' $\mathrm{D}$ value shows the same growth tendency with GDP, and it is tending to 0.4 in 2015 . Comparing with DMC, the decoupling condition of MF is quite different. During 1997-2015, only biomass and non-metallic minerals are absolutely decoupled, and their decoupling condition maintained a high level. However, fossil fuels and metal ores are not decoupled with GDP during 1990-2013. In 2014, metal ores' $\mathrm{D}$ value is increasing to 0.5 and achieves a relative decoupling finally.

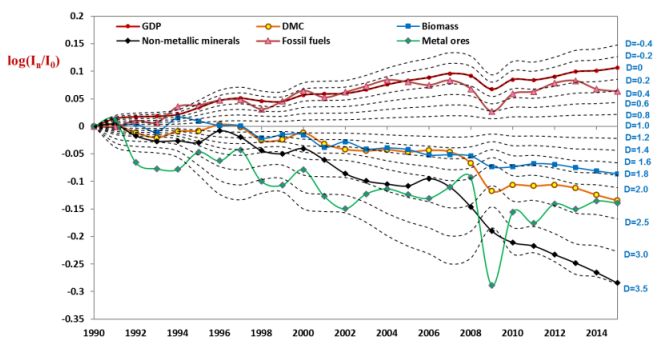

Figure 9. The decoupling trend of DMC in Japan, 1990-2015.

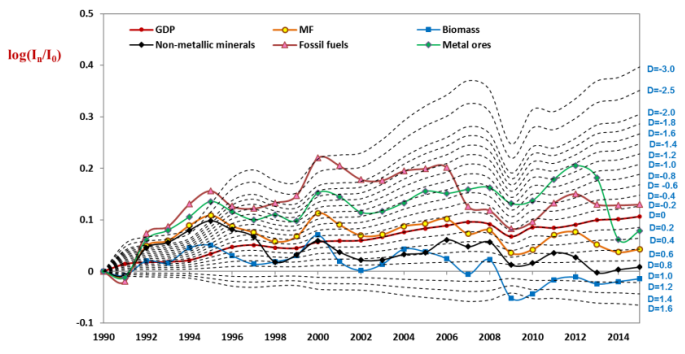

Figure 10. The decoupling trend of MF in Japan, 1990-2015. 
Fig. 11 and Fig. 12 show the decoupling results of resources' DMC and MF in the United States during 1990-2015, respectively. The condition of DMC is absolute decoupling, and metal ores and non-metallic minerals have also achieved an absolute decoupling since 2009. The characteristics of United States' DMC is that metal ores are the best resources in decoupling condition and biomass is the worst. From the MF viewpoint, the condition of resource use is relative decoupling. Non-metallic minerals are the best resource whose $\mathrm{D}$ value is 0.6 in 2015 , and biomass is the worst whose $\mathrm{D}$ value is 0.2 .

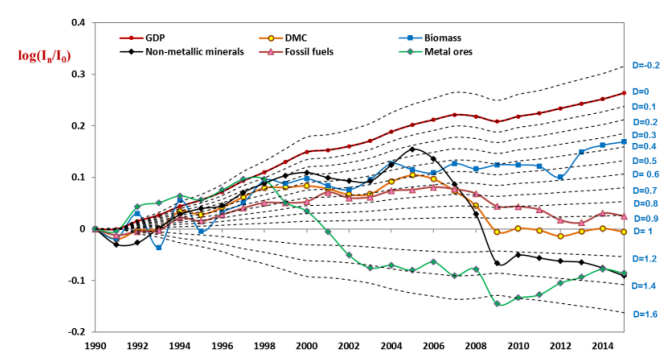

Figure 11. The decoupling trend of DMC in the United States, 19902015.

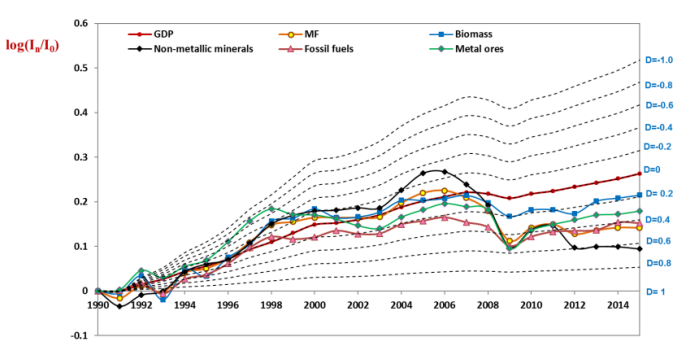

Figure 12. The decoupling trend of MF in the United States, 1990-

2015.

\section{Discussion}

From the viewpoint of countries, three developed countries have enjoyed much better decoupling condition than developing countries. Six countries' decoupling condition shows different characteristics and tendency. In addition, their decoupling conditions are closely related to the development strategy and stage.

For developing countries, China's GDP and resource use is increasing smoothly and all kinds of resources are decoupled from GDP basically during 1990-2015. That's due to their stable and continuous environmental policy and their increasing industrial technology. For Brazil, its' three kinds of resources' DMC and MF are not decoupled with GDP in during 1990-2015. That means Brazil's economic growth is closed related to resource use, especially raw resource (metal ores enjoy the only decoupling condition). India's decoupling condition is similar to China, but their metal ores' DMC is not decoupled with GDP. That means India's social development is also stable, but their industrial technology needs to improve and international trade policies are to be considered.

For developed countries, all kinds of resources' DMC and MF in Australia are relative decoupling in 2015, and their decoupling condition of MF is better than DMC, because Australia exports a lot of resources to other countries. Japan as the most successful decoupling country in our study, its' three kinds of resources' DMC is absolute decoupling in 2015. Facing to the resources' scarce condition, Japan's environmental policy is successful and strict. In 2015, all kinds of DMC and MF are decoupled in the United States. The reason is that the geographical conditions and natural resources distribution are superior, and they are already in the stage of ecological restoration in the late industrialization expressed by the environmental Kuznet's curve.

By comparing the decoupling results of six countries' DMC and MF, we find that the developing countries' decoupling condition of MF is better than that of DMC, while the developed countries' decoupling condition is the opposite. The previous study by Wiedmann shows a similar result [11]. The reason for this condition is that developing countries usually produce a lot of products which will be consumed in developed countries through world trade network [12]. For developed countries, they are the opposite, because they usually consume a lot of resources which are produced by developing countries. Because the MF indicator not only considers the resource in domestic consumption but also includes the part of world trade, it's a more comprehensive and scientific indicator than when we analyze different countries' decoupling condition. 


\section{Conclusions}

1. The developed countries' decoupling condition is better than developing countries generally. Brazil's decoupling condition is the worst while Japan's decoupling policy is most successful.

2. The developing countries' decoupling condition of MF is better than that of DMC, while the developed countries' decoupling condition of DMC is better than that of MF.

3. Our findings emphasize the importance of choosing MF indicators when analyzing decoupling policy.

\section{Acknowledgements}

This research was financially supported by the National Natural Science Foundation of China (41401636, 71403175, 51474067 and 71373003), and Fundamental Research Funds for the Central Universities (N172504026).

\section{References}

[1] H. Wang, S. Hashimoto, Y. Moriguchi, Q. Yue, Z. Lu. Resource Use in Growing China. Journal of Industrial Ecology. 16(2012) 481-492.

[2] F. Ma, H. Wang, B. Zhu, D. Chen, H. Dai, J. Wang, S. Zhao, Q. Yue, G. Zhang, Y. Xie, Y. Geng, T. Du. Material footprint of a fast-industrializing region in China, Part 1: Exploring the materialization process of Liaoning Province. Resources, Conservation and Recycling. 134(2018) 228-238.

[3] Q. Yue, H. Wang, C. Gao, T. Du, M. Li, Z. Lu. Analysis of iron in-use stocks in China. Resources Policy. 49(2016) 315-322.

[4] Q. Yue, H. Wang, Z. Lu, S. Zhi. Analysis of anthropogenic aluminum cycle in China. Transactions of Nonferrous Metals Society of China. 24(2014) 1134-1144.

[5] H. Wang, H. Dai, L. Dong, Y. Xie, Y. Geng, Q. Yue, F. Ma, J. Wang, T. Du. Co-benefit of carbon mitigation on resource use in China. Journal of Cleaner Production. 174(2018) 1096-1113.

[6] Z. Lu, H. Wang, Q. Yue. Decoupling Analysis of the Environmental Mountain-with Case Studies from China. Journal of Industrial Ecology. 19(2015) 1082-1090.

[7] UNEP. Decoupling natural resource use and environmental impacts from economic growth, A Report of the Working Group on Decoupling to the International Resource Panel. 2011. Fischer-Kowalski, M., M. Swilling, E.U. von Weizsäcker, Y. Ren, Y. Moriguchi, W. Crane, F. Krausmann, N. Eisenmenger, S. Giljum, P. Hennicke, P. Romero Lankao, and A. Siriban Manalang.

[8] H. Wang, S. Hashimoto, Q. Yue, Y. Moriguchi, Z. Lu. Decoupling Analysis of Four Selected Countries. Journal of Industrial Ecology. 17(2013) 618-629.

[9] H. Schandl, J. West. Resource use and resource efficiency in the Asia-Pacific region. Global Environmental Change-Human and Policy Dimensions. 20(2010) 636-647.

[10] Global Material Flows Database. http://www.resourcepanel.org/global-material-flows-database.

[11] T. O. Wiedmann, H. Schandl, M. Lenzen, D. Moran, S. Suh, J. West, and K. Kanemoto. The material footprint of nations. Proceedings of the National Academy of Sciences of the United States of America. 112(2015) 6271-6276.

[12] T. O. Wiedmann and M. Lenzen. Environmental and social footprints of international trade. Nature Geoscience. 11(2018) 314-321. 